\title{
ABANDONO DO ATENDIMENTO EM UMA CLÍNICA-ESCOLA DE PSICOLOGIA INFANTIL: VARIÁVEIS ASSOCIADAS
}

\author{
Carina Cella Panaia Mantovani* \\ Edna Maria Marturano \\ Edwiges Ferreira de Mattos Silvares"
}

\begin{abstract}
RESUMO. O objetivo deste estudo retrospectivo foi levantar taxas de desistência e abandono do atendimento em uma clínica-escola de psicologia infantil e identificar fatores de risco sócio-demográficos, clínicos e familiares para ambos os desfechos. Dados de 258 crianças foram obtidos nos prontuários. Desistiram do atendimento logo após a entrevista inicial $16 \%$ das famílias. Entre as famílias não desistentes, $49 \%$ interromperam o atendimento antes da alta. Foram comparados, quanto aos fatores de risco, os grupos desistente e não desistente. Além disso, no grupo não desistente, os subgrupos abandono (clientes que compareceram ao menos uma vez antes de interromperem o tratamento) e alta clínica (clientes que persistiram até o final do atendimento) também foram comparados. Famílias desistentes apresentaram mais crianças nas séries iniciais, com menos problemas de comportamento. $\mathrm{O}$ abandono foi associado a menor recurso cognitivo e menor idade da criança, pai mais jovem e maior exposição da família a estresse psicossocial..
\end{abstract}

Palavras-chave: Tratamento de crianças; desistência; abandono.

\section{DROPOUT IN A SCHOOL-CLINIC OF CHILD PSYCHOLOGY}

\begin{abstract}
This retrospective study aimed at investigating the rates of treatment nonattendance and dropout at a universityaffiliated child guidance clinic and identifying socio demographic, clinical and family risk factors that contribute to them. We obtained archival data of 258 children. Sixteen percent of the families did not return after their intake interview. Among families that returned, forty-nine percent dropped out of treatment. Groups were compared with respect to risk factors for selftermination: nonattenders (clients who did not return after intake) versus attenders (clients who returned); dropouts (clients that attended at least one session before interrupting treatment) versus completers (clients that persisted until treatment conclusion). Children from nonnattender families attended earlier school grades and had less behavior problems. Children in the dropout group had lower scores in intelligence assessment, their fathers were younger and their families were more exposed to psychosocial stress, as compared to completers.
\end{abstract}

Key words: Child treatment; nonattendance; dropout.

\section{ABANDONO EN UNA CLÍNICA-ESCUELA DE PSICOLOGÍA INFANTIL}

RESUMEN. El objetivo fue buscar tasas de retirada y abandono del tratamiento en una clínica-escuela de psicología para niños y identificar factores de riesgo demográficos, clínicos y ambientales para ambos los desenlaces. Se utilizaron datos de archivo de 258 niños. No llegaran a iniciar el tratamiento en seguida a la primera entrevista 16\% de las familias. Entre las que retornaran, $49 \%$ terminaran el tratamiento prematuramente. Se compararan las familias que no regresaran después de la entrevista inicial con las familias que retornaran. Las familias que abandonaran el tratamiento empezado fueran comparadas con las que persistieran hasta el fin. En las familias que no iniciaran en tratamiento los niños tenían menos problemas de conducta y cursaban años iniciales en la escuela. En las familias que abandonaran, los niños eran más jóvenes, tuvieran peor desempeño en test cognitivo, el estatus educativo de los padres era menor y la familia estaba más expuesta a estrés psicosocial.

Palabras-clave: Tratamiento de niños; retirada; abandono.

\footnotetext{
Mestre em Ciências Médicas (Saúde Mental) pela Faculdade de Medicina de Ribeirão Preto da Universidade de São Paulo, Brasil.

\# Doutora em Ciências - Psicologia. Professora titular da Universidade de São Paulo, Brasil.

II Doutora em Psicologia Experimental. Professora titular da Universidade de São Paulo, Brasil.
} 
A literatura indica que a demanda por atendimento psicológico para crianças supera a oferta (Vanni \& Maggi, 2005), particularmente nos países em desenvolvimento (Lai, Pang, Wong, Lum \& Lo, 1998), porém 28 a $75 \%$ das famílias que conseguem atendimento não chegam a iniciá-lo ou então o abandonam em algum momento (Campezzato \& Nunes, 2007; Lhullier, Nunes \& Horta, 2006; Luk et al., 2001; Sirles, 1990). As taxas de abandono tendem a ser maiores em estudos que focalizam a prática clínica de rotina, em comparação com estudos controlados de avaliação de tratamentos (Luk et al., 2001). Assim, é preciso considerar, como apontam Harpaz-Rotem, Leslie e Rosenheck (2004), que o acesso da criança ao atendimento não é garantia de permanência no mesmo. As famílias podem interromper o atendimento em qualquer fase, e o término em diferentes momentos parece estar associado a diferentes fatores (Lai et al., 1998; Luk et al., 2001).

No Brasil, a faixa etária de zero a 12-14 anos constitui o segmento mais numeroso da clientela que busca atendimento em clínicas-escola de psicologia (Lhullier et al., 2006; Romaro \& Capitão, 2003). Na clientela infantil, Marturano, Degani, Alves e Miranda (1993) encontraram taxa de abandono igual a $23 \%$, ao passo que Romaro e Capitão (2003) obtiveram taxa de adesão de $72 \%$, o que corresponde a um percentual de $28 \%$ de abandono.

Altas taxas de abandono têm implicações importantes. Em primeiro lugar, o abandono do atendimento tem impacto na vida do indivíduo que o busca, pois não permite que este receba os benefícios do tratamento. Pesquisas mostram que há ganhos significativos para as crianças e famílias que completam um tratamento psicológico, em relação às que o interrompem (Boggs et al., 2004). Em segundo lugar, perdas de casos contribuem para reduzir o número de pessoas que efetivamente recebem cuidados. Além disso, o término prematuro frequientemente é precedido por faltas e por cancelamentos de retornos agendados (Lhullier et al., 2006; Marturano et al., 1993), sinalizando ocupação não aproveitada de uma vaga que poderia ser oferecida para outras famílias. Como resultado, a produtividade da equipe clínica decresce e o custo dos serviços aumenta (Kazdin, Holland \& Crowley, 1997). Por isso, deve-se buscar a compreensão dos fatores relacionados ao abandono, de modo que se possam otimizar os recursos disponíveis, aumentando a probabilidade de retenção das famílias no atendimento.

O abandono do atendimento pelas famílias tem sido associado a variáveis sócio-demográficas (Wierzbicki \& Pekarik, 1993), assim como a variáveis clínicas (Dierker, Nargiso, Wiseman \& Hoff, 2001), institucionais (Lhullier et al., 2006) e da relação terapêutica (Harwood \& Eyberg, 2004). Os fatores sócio-demográficos são os mais investigados, tendo sido encontrada associação entre abandono e fatores como: mães jovens, famílias monoparentais (Campbell, Baker \& Bratton, 2000; Kazdin et al., 1997), grupo minoritário, famílias em desvantagem sócio-econômica (Kazdin, 1994; Kazdin, Stolar \& Marciano, 1995; Kazdin et al., 1997) e baixa escolaridade materna (Luk et al., 2001; Sirles, 1990).

Entre as variáveis clínicas da criança, a história antisocial (Kazdin et al., 1997; Luk et al., 2001) e a presença de queixas múltiplas têm sido associadas positivamente ao abandono (Dierker et al., 2001). Por outro lado, a intensidade dos sintomas de ansiedade e a severidade do transtorno têm associação negativa com abandono (Harpaz-Rotem et al., 2004; Kendall \& Sugarman, 1997).

No sistema familiar, têm sido apontados como preditores de abandono do atendimento o alto nível de estresse nos eventos de vida (Kazdin, 1994; Prinz \& Miller, 1994), assim como as práticas educativas coercitivas e maus tratos (Kazdin et al., 1997; Lau \& Weizs, 2003). As atitudes e expectativas dos pais frente ao atendimento, a percepção de barreiras ao tratamento e problemas na relação terapêutica são variáveis da relação família-clínica que afetam a disposição das famílias para persistir no tratamento ou interrompê-lo (Attride-Stirling, Davis, Farrell, \& Day, 2004; Boggs et al., 2004; Garcia \& Weisz, 2002; Kazdin et al., 1997; Luk et al., 2001; Prinz \& Miller, 1994).

Em relação ao funcionamento institucional, o intervalo de tempo entre a avaliação diagnóstica e o início do tratamento tem sido associado ao auto-desligamento (Kendall \& Sugarman, 1997; Lhullier et al., 2006; Marturano et al., 1993). Kendall e Sugerman (1997) encontraram maiores taxas de desistência e abandono entre as famílias que esperaram oito semanas para iniciar a intervenção, quando comparadas às famílias admitidas ao tratamento logo em seguida ao diagnóstico. Em dois estudos brasileiros, o intervalo entre a avaliação diagnóstica e o tratamento, dividido na marca de 30 dias, mostrou associação direta seja com desistência na fila de espera (Marturano et al., 1993) ou com abandono do tratamento (Lhullier et al., 2006).

Apesar da diversidade de fatores que têm sido associados ao abandono, poucas tendências consistentes são encontradas. A meta-análise conduzida por Wierzbicki e Pekarik (1993) sobre 125 estudos, considerando variáveis demográficas, psicológicas e do terapeuta, detectou consistência nos resultados somente para preditores demográficos, como grau de instrução e renda. Em revisão de 24 artigos publicados desde 1990, com foco no abandono do tratamento psicológico de crianças, as autoras deste artigo identificaram apenas 
preditores socioeconômicos e de stress parental. Há indícios de que, em comparação com estudos conduzidos em serviços de saúde mental da comunidade, pesquisas feitas em clínicas ligadas a universidades, trabalhando com amostras mais homogêneas quanto ao diagnóstico e focalizando tratamento específico, tendem a relatar mais resultados significativos na predição de abandono (Pina, Silverman, Weens, Kurtines \& Goldman, 2003).

Os estudos sobre abandono não são facilmente comparáveis entre si, devido a diferenças de método, em itens como definição de abandono, indistinção entre abandono em diferentes fases do atendimento, seleção e definição de preditores de abandono. Um aspecto muito importante a ser considerado é a falta de uniformidade no uso do termo abandono, dificultando a comparação entre resultados de diferentes pesquisas (Kazdin et al., 1995). O abandono de um atendimento, conforme Lai et al. (1998) e Luk et al. (2001), corresponde ao término prematuro da terapia, por parte dos pais, sem o consentimento clínico. Esses autores ainda consideram que o estágio em que o abandono ocorre também deve ser observado: aqueles que faltam ao retorno depois da entrevista inicial de avaliação formam o grupo de abandono precoce; aqueles que fazem o seguimento ao menos uma vez antes de abandonarem formam o grupo de abandono tardio. A mesma distinção é adotada neste artigo, usando-se o termo desistência para abandono precoce e simplesmente abandono para abandono tardio. A escolha dos termos foi feita para maior clareza na distinção entre o não início de uma intervenção prescrita e a interrupção de uma intervenção implementada. Assim, desistência significa renúncia ao tratamento antes mesmo de ele começar, enquanto abandono significa interrupção do tratamento já iniciado. Distinção semelhante foi feita previamente por Dierker et al. (2001).

O foco do estudo é o abandono das famílias em uma clínica de psicologia que atende crianças com queixa escolar. Em clínicas brasileiras que oferecem serviços gratuitos de saúde mental, a queixa escolar é um dos principais motivos de procura de atendimentos para crianças e adolescentes (Braga \& Morais, 2007; Romaro \& Capitão, 2003; Schoen-Ferreira, Silva, Aznar Farias \& Silvares, 2002). Há indícios de co-ocorrência de problemas emocionais / de comportamento nessa clientela, da ordem de 60 a 74\% (Betti, 2007; D'Abreu, 2008).

Com o reconhecimento crescente de que crianças apresentando simultaneamente baixo desempenho escolar e social devem ser alvo de atenção psicológica (Del Prette $\&$ Del Prette, 2005), o que nem sempre é possível no âmbito da escola, modalidades de apoio psicopedagógico têm sido propostas, implementadas e avaliadas na rede de saúde, com foco na queixa escolar (Elias \& Marturano,
2005). É nesse contexto que se inscreve o presente estudo, em que, diferentemente das pesquisas com foco no abandono de psicoterapia, buscam-se informações sobre abandono do atendimento psicopedagógico.

Os objetivos do trabalho foram primeiramente levantar as taxas de desistência e de abandono e, a partir disso, identificar fatores de risco sócio-demográficos, clínicos, de exposição a estressores e da relação famíliaclínica, tanto para desistência, quanto para o abandono do atendimento.

\section{MÉTODO}

\section{Contexto da pesquisa}

A pesquisa foi aprovada pelo Comitê de Ética em Pesquisa do Hospital das Clinicas da FMRP-USP.

A investigação foi conduzida em uma clínicaescola de psicologia de um hospital universitário vinculado ao Sistema Único de Saúde - SUS, em uma cidade de aproximadamente 500 mil habitantes no interior do Estado de São Paulo. O atendimento à clientela da clínica é gratuito, feito predominantemente por profissionais recém-formados, como parte de um programa de aprimoramento profissional, sob a supervisão de psicólogos docentes ou membros da equipe técnica. Estes últimos fazem o acolhimento das famílias na $1^{a}$ consulta. Pósgraduandos eventualmente conduzem projetos de intervenção na clínica.

A clínica atende crianças de 6 a 11 anos com queixa escolar, encaminhadas com guia de referência do SUS. Na abordagem da queixa escolar, adota-se uma perspectiva desenvolvimentista, com ênfase nos conceitos de tarefa de desenvolvimento, resiliência, competência, recursos, mecanismos de risco, proteção e vulnerabilidade.

Na primeira consulta, destinada à triagem clínica e ao acolhimento da família, um dos psicólogos da equipe técnica permanente realiza uma entrevista com a mãe ou responsável e uma sondagem com a criança. A entrevista tem foco no esclarecimento da queixa e na identificação de recursos e mecanismos de risco / proteção pessoais e ambientais na história de vida da criança. Na sessão com a criança, faz-se uma sondagem do potencial intelectual, do repertório de leitura e escrita e do comportamento frente a tarefas de cunho acadêmico.

Logo em seguida à entrevista e à sondagem, o psicólogo faz uma devolução à família, com base nas informações colhidas. Na devolução se apresentam os esclarecimentos, indicações, recomendações e encaminhamentos cabíveis. Quando o caso se 
enquadra nos critérios de admissão e as informações colhidas indicam que a criança pode se beneficiar do atendimento psicopedagógico, expõe-se o funcionamento da clínica, oferece-se atendimento e a mãe é convidada a participar, enquanto aguarda vaga, de um programa informativo pré-intervenção oferecido no ambulatório, com seis encontros semanais, denominado "Como ajudar seu filho na escola".

Posteriormente, a família é convocada, por carta ou telefone, para o $1^{\circ}$ retorno. Neste se estabelece um contrato verbal, válido até o final do semestre escolar, que pode ser renovado. $\mathrm{O}$ atendimento consiste de apoio psicopedagógico à criança, individual ou em grupo, em esquema semanal. Uma vez iniciado este atendimento, contatos com a família são agendados com periodicidade variável, para troca de informações e para orientação, ou sempre que solicitado pela própria família. O contato com a escola é decidido caso a caso.

A cada final de semestre escolar a criança é reavaliada. Com base nos registros de evolução do atendimento e nessa reavaliação, o profissional responsável e o supervisor definem uma conduta - alta ou renovação do contrato por mais um semestre - e esta é submetida ao familiar que acompanha a criança, para uma decisão final por consenso.

De acordo com a perspectiva desenvolvimentista adotada, o atendimento psicopedagógico tem por objetivo ativar operações e estratégias cognitivas, bem como processos de adaptação afetivo-social, relacionados às tarefas de desenvolvimento da fase escolar. Entre os objetivos específicos do atendimento estão a ativação da curiosidade, o fortalecimento da motivação intrínseca para a aprendizagem, o desenvolvimento de um positivo senso de autoeficácia para tarefas escolares e o domínio de estratégias para o aprendizado de leitura e escrita. Busca-se alcançar os objetivos mediante a exposição da criança a mini-situações de experiência de aprendizagem mediada, individuais ou em grupo. $\mathrm{O}$ enquadramento é situacional, pois se baseia no que ocorre durante as sessões, e operativo, na medida em que a relação entre a criança e o profissional é estabelecida em torno de tarefas precisas e concretas, compromissadas com o aprender. Uma descrição e avaliação desta modalidade de atendimento pode ser encontrada em Elias e Marturano (2005).

\section{Fontes de dados}

As fontes de dados foram registros e prontuários referentes a crianças que passaram pela primeira consulta na clínica, nos anos de 1999 a 2002. Nesse período, o ano de 1999 se diferenciou dos demais porque nele foi feito o recrutamento de clientes para dois programas de intervenção com finalidade de pesquisa. Crianças encaminhadas a outros serviços ou que tiveram seus casos resolvidos na primeira consulta foram excluídas da pesquisa, assim como as que se encontravam em atendimento por ocasião do levantamento dos casos elegíveis para o estudo. A amostra do estudo foi composta por 258 crianças (183 meninos e 75 meninas).

\section{Instrumentos}

Os dados foram obtidos no livro de registro de atendimentos do ambulatório, nas fichas de inscrição e nos prontuários dos clientes; nos prontuários, foram consultados os protocolos dos instrumentos aplicados rotineiramente no serviço, por ocasião da $1^{\text {a }}$ consulta (Betti, 2007): Teste de Matrizes Progressivas Coloridas de Raven - Escala Especial; Escala Comportamental Infantil A-2 para pais - ECI; Escala de Eventos Adversos - EEA.

\section{Procedimento}

Informações sobre data da $1^{\mathrm{a}}$ consulta, data do $1^{\mathrm{o}}$ retorno e data da alta foram obtidas no livro de registro de atendimentos. $\mathrm{Na}$ ficha de inscrição foram levantadas as variáveis sócio-demográficas: idade e escolaridade da criança, idade e escolaridade dos pais, composição familiar (família nuclear biológica, família monoparental, família recasada). Dos prontuários foram extraídas informações sobre nível intelectual (Raven), problemas de comportamento (ECI) e estresse (EEA). A avaliação da exposição da criança e da família a estressores, a partir da EEA, baseou-se nos seguintes indicadores de estresse nos últimos 12 meses: instabilidade financeira; adversidade na relação do casal parental; eventos adversos na vida pessoal da criança; eventos adversos na vida escolar da criança; total de eventos adversos recentes.

A fim de levantar as taxas de desistência e abandono do atendimento e as variáveis relacionadas a elas, três condições de encerramento do atendimento foram consideradas: (1) alta clínica, que consistiu no seguimento da intervenção até seu encerramento, decidido mediante consenso entre o profissional encarregado do atendimento e os responsáveis pela criança; (2) desistência, entendida como o não comparecimento à intervenção prescrita, antes de esta ser iniciada, por decisão unilateral dos pais ou responsáveis, sem a concordância do profissional; (3) abandono, ou seja, interrupção unilateral do atendimento, por parte dos pais ou responsáveis, sem a 
concordância do profissional, depois de iniciada a intervenção.

De acordo com as condições de encerramento, foram formados os seguintes grupos: Grupo Desistente, que não chegou a iniciar a intervenção; Grupo Não Desistente, que iniciou a intervenção, subdividido em Grupo Abandono, com as crianças cujas famílias interromperam o atendimento durante a intervenção, e Grupo Alta Clínica, com crianças que passaram por toda a intervenção e obtiveram alta.

Foram feitas análises estatísticas no programa SPSS v. 13 para identificar condições de risco para desistência, comparando-se famílias desistentes com famílias não desistentes; e condições de risco para abandono, comparando-se, entre as não desistentes, o Grupo Abandono com o Grupo Alta Cínica. As análises são descritas na seção Resultados.

\section{RESULTADOS}

Das crianças admitidas na clinica, $76 \%$ apresentavam sintomas comportamentais indicativos de necessidade de atendimento psicológico, segundo critério da ECI, com predomínio de comportamentos de externalização (fica mal humorado e nervoso; impaciente e irrequieto; irritável; muito agitado; muitas vezes é desobediente), mas também com sintomas emocionais (agarrado à mãe, inseguro).

Foram encontradas 41 famílias desistentes (Grupo Desistente), 107 que abandonaram o tratamento (Grupo Abandono) e 110 que persistiram até a alta
(Grupo Alta Clínica). De acordo com essa distribuição, observa-se que $16 \%$ das famílias desistiram do atendimento antes do início da intervenção. O tempo de espera entre a $1^{\text {a }}$ consulta e o $1^{\circ}$ retorno foi associado com desistência: $72 \%$ dos não desistentes e $45 \%$ dos desistentes tiveram retorno agendado dentro de três meses a contar da $1^{\mathrm{a}}$ consulta, ou seja, houve mais famílias desistentes entre aquelas que tiveram de esperar mais de três meses pelo $1^{\circ}$ retorno $\left(\mathrm{X}^{2}=8,80, \mathrm{p}=0,003\right)$.

Entre as famílias não desistentes, $51 \%$ persistiram no atendimento até a alta clínica. As taxas de abandono variaram de acordo com o ano da $1^{\mathrm{a}}$ consulta. No ano de 1999, em que houve a seleção de participantes para dois programas de intervenção com finalidade de pesquisa, a taxa de abandono foi igual a $39 \%$, ao passo que nos anos subseqüentes, em que não houve pesquisas, foi de $54 \%$, (X2 = 4,278, p = 0,039), variando entre $51 \%$ e $59 \%$. Mediante esse resultado, os dados de 1999 foram excluídos das comparações subseqüentes entre as condições de abandono e alta clínica.

Antes da análise estatística, foi verificada a normalidade da distribuição dos dados; o teste Kolmogorov-Smirnov indicou que apenas a variável idade tinha distribuição normal. As comparações de grupo foram então efetivadas por meio do teste $U$ de Mann-Whitney, ou do Teste Exato de Fisher no caso de variáveis categóricas.

$\mathrm{Na}$ Tabela 1 são apresentados os resultados das comparações entre famílias desistentes $(n=41)$ e não desistentes $(\mathrm{n}=217)$.

Tabela 1. Escores Médios nas Variáveis Sociodemográficas e Clínicas Avaliadas na $1^{\text {a }}$ Consulta, nos Grupos Desistente e Não Desistente.

\begin{tabular}{lccc}
\hline \multicolumn{1}{c}{ Variável } & $\begin{array}{c}\text { Desistente } \\
(\mathbf{n = 4 1 )}\end{array}$ & $\begin{array}{c}\text { Não Desistente } \\
(\mathbf{n = 2 1 7})\end{array}$ & Z \\
\hline Idade da criança & 9,10 & 9,29 & 0,89 \\
Escolaridade da criança & 2,15 & 2,64 & $2,08^{\text {a }}$ \\
Idade da mãe (em anos) & 35,24 & 35,32 & 0,94 \\
Idade do pai (em anos) & 37,78 & 37,66 & 0,64 \\
Escolaridade da mãe (em anos completos) & 6,98 & 6,92 & 0,27 \\
Escolaridade do pai (em anos completos) & 6,45 & 6,21 & 0,98 \\
Nível intelectual - percentil Raven & 41,95 & 36,97 & 0,85 \\
Problemas de comportamento (escore total ECI) & 19,92 & 23,40 & $2,45^{\text {b }}$ \\
Instabilidade financeira (itens EEA) & 0,63 & 0,49 & 0,57 \\
Adversidade na relação do casal parental (itens EEA) & 0,35 & 0,26 & $1,33^{*}$ \\
Eventos adversos na vida pessoal da criança (itens EEA) & 0,00 & 0,03 & 1,07 \\
Eventos adversos na vida escolar da criança (itens EEA) & 0,66 & 0,63 & 0,97 \\
Eventos adversos nos últimos 12 meses (escore total EEA) & 2,73 & 2,36 & 0,24 \\
\hline
\end{tabular}

${ }^{a} \mathrm{p} \leq 0,05 ;{ }^{b} \mathrm{p} \leq 0,01 ; * \mathrm{*}<0,10$ 
Como mostra a Tabela 1, na comparação entre os grupos Desistente e Não Desistente observam-se duas diferenças significativas. As crianças do Grupo Desistente são menos escolarizadas e apresentam menos problemas de comportamento. Resultados não incluídos na tabela indicaram, segundo o Teste U, que quatro itens da ECI diferenciam os grupos, com menor freqüência ou intensidade no Grupo Desistente: tem dor de estômago ou vômito; é uma criança impaciente, irrequieta; muitas vezes destrói suas próprias coisas ou dos outros; muitas vezes é desobediente.

Uma diferença marginalmente significativa $(\mathrm{p}<$ 0,10 ) assinala tendência à maior adversidade na relação do casal parental nas famílias desistentes.

Os resultados das comparações entre os grupos Abandono e Alta Clínica são apresentados na Tabela 2.

Tabela 2. Escores Médios nas Variáveis Sociodemográficas e Clínicas Avaliadas na 1ª Consulta, nos Grupos Abandono e Alta Clínica.

\begin{tabular}{|c|c|c|c|}
\hline Variável & $\begin{array}{c}\text { Abandono } \\
2000-2002 \\
(n=81)\end{array}$ & $\begin{array}{c}\text { Alta } \\
2000-2002 \\
(n=69)\end{array}$ & $\mathbf{Z}$ \\
\hline Idade da criança & 8,96 & 9,38 & $1,98^{\mathrm{a}}$ \\
\hline Escolaridade da criança & 2,30 & 2,67 & $2,18^{\mathrm{a}}$ \\
\hline Idade da mãe (em anos) & 34,22 & 35,89 & $1,53^{*}$ \\
\hline Idade do pai (em anos) & 36,11 & 38,12 & $2,17^{\mathrm{a}}$ \\
\hline Escolaridade da mãe (em anos completos) & 6,58 & 7,35 & $1,37 *$ \\
\hline Escolaridade do pai (em anos completos) & 5,94 & 7,17 & $1,51 *$ \\
\hline Nível intelectual - percentil Raven & 31,72 & 40,39 & $2,14^{\mathrm{a}}$ \\
\hline Problemas de comportamento (escore total ECI) & 22,70 & 22,12 & 0,70 \\
\hline Instabilidade financeira (itens EEA) & 0,53 & 0,30 & $2,01^{\mathrm{a}}$ \\
\hline Adversidade na relação do casal parental (itens EEA) & 0,38 & 0,13 & $1,96^{\mathrm{a}}$ \\
\hline Eventos adversos na vida pessoal da criança (itens EEA) & 0,00 & 0,05 & $1,86^{*}$ \\
\hline Eventos adversos na vida escolar da criança (itens EEA) & 0,74 & 0,82 & 0,89 \\
\hline Eventos adversos nos últimos 12 meses (escore total EEA) & 2,75 & 1,87 & $2,53^{\mathrm{b}}$ \\
\hline
\end{tabular}

${ }^{\mathrm{a}} \mathrm{p} \leq 0,05 ;{ }^{\mathrm{b}} \mathrm{p} \leq 0,01 ; * \mathrm{p}<0,10$

Ao se compararem os grupos Abandono e Alta Clínica, aparecem diferenças significativas em sete variáveis. As crianças do Grupo Abandono são mais jovens, assim como seus pais. Elas apresentam ainda média mais baixa no indicador de nível intelectual. Por ocasião da $1^{a}$ consulta, as famílias deste grupo relatam maior estresse psicossocial recente, notadamente instabilidade financeira e estressores intra-familiares. A instabilidade financeira inclui situações como perda de emprego de um dos pais e redução da renda familiar. As adversidades parentais englobam situações tais como aumento de conflitos entre os pais, separação e divórcio. Uma diferença marginalmente significativa foi encontrada no número de eventos adversos na vida pessoal da criança, como hospitalização, enfermidade grave e acidente com seqüela, indicando maior presença de tais situações no Grupo Alta Clínica. O exame das médias desta variável nas Tabelas 1 e 2 indica que sua ocorrência é baixa em todos os grupos, mas sempre maior no grupo que persistiu no tratamento até a alta.

Nas comparações entre grupos envolvendo a composição familiar, não foram encontradas diferenças entre os grupos.

\section{DISCUSSÃO}

Dos 258 casos analisados, $16 \%$ desistiram do atendimento antes da intervenção; confirmou-se como um fator de risco para desistência o tempo na fila de espera para o tratamento, tal como relatado por Kendall e Sugarman (1997). Na comparação entre os grupos Desistente e Não Desistente, o grupo Desistente apresentou maior número de crianças nas séries iniciais e crianças com menor freqüência de problemas de comportamento. A menor severidade dos problemas de comportamento mostrou-se um preditor de desistência, condição também encontrada por Sirles (1990) em seu estudo em uma clínica de psiquiatria infantil ligada a uma faculdade de medicina. Harpaz-Rotem et al (2004) verificaram que o abandono precoce (nos primeiros 30 dias) estava associado a menor severidade clínica dos problemas da criança. $\mathrm{Na}$ presente investigação, a conjugação entre a menor severidade dos sintomas comportamentais e a menor cronicidade do problema escolar pode ter levado os pais a suporem que as possíveis dificuldades das crianças não eram suficientes para demandar atendimento especializado; o prolongado tempo de espera pode ter sido um 
potencializador dessa disposição, contribuindo para a desistência. O risco de desistência associado ao tempo de espera também confirma dados da literatura, indicando que o tempo de espera entre o diagnóstico e o início do tratamento é um preditor de abandono (Kendall \& Sugarman, 1997; Lhullier et al, 2006).

Entre as famílias que iniciaram a intervenção, a taxa de abandono de $49 \%$ foi semelhante à taxa de alta clínica, de $51 \%$. Em relação ao total de casos analisados, o percentual de abandono foi de $42 \%$. Esta taxa assemelha-se a taxas encontradas em estudos sobre abandono realizados em serviços da comunidade (Campbell et al., 2000; Garcia \& Weizs, 2002; Harpaz-Rotem et al., 2004, Luk et al., 2001) e contrasta com as taxas encontradas em clínicas vinculadas a universidades, em geral mais baixas (Kazdin, 1994; Kendall \& Sugarman, 1997; Lai et al., 1998; Pina et al., 2003; Prinz \& Miller, 1994; Romaro \& Capitão, 2003).

Ao se comparar o período em que houve exclusivamente o atendimento de rotina da clínica (2000-2002) com o período em que foram conduzidos projetos de intervenção com finalidade de pesquisa (1999), a taxa de abandono é significativamente menor neste último. Esse resultado é concordante com a observação de Luk et al. (2001), de que estudos controlados de avaliação de tratamentos tendem a relatar taxas menores de abandono. Quando se desenvolvem projetos de intervenção, há um maior controle das condições de atendimento, de modo a garantir a fidelidade da implementação da intervenção e maximizar as chances de efeitos positivos. Os terapeutas são mais bem treinados e, como lembram Luk et al. (2001), freqüentemente mais entusiasmados em relação ao ensaio de pesquisa. Com frequiência se estabelecem procedimentos para maximizar a adesão da família. Esses fatores provavelmente concorrem para os menores índices de abandono relatados em estudos de avaliação da eficácia ou efetividade de intervenções (por exemplo, Lai et al., 1998; Pina et al., 2003), quando comparados a índices obtidos na rotina de serviços de saúde mental (por exemplo, Campbell et al., 2000; Dierker et al., 2001; Garcia \& Weisz, 2002).

Outra explicação para essa discrepância entre taxas de abandono no atendimento de rotina e no período em que foram conduzidos projetos de intervenção com finalidade de pesquisa pode ser proposta. É que, em estudos controlados de avaliação de tratamentos, geralmente o contrato com a família é de tempo limitado, com uma clara especificação de seu término, ao passo que na rotina dos serviços de saúde mental a duração do tratamento é indefinida, embora no serviço investigado haja uma prática de contratos semestrais. Segundo Kendall e Sugarman (1997), dados de pesquisa em psicoterapia de adultos sugerem que as taxas de atrito podem ser reduzidas quando os clientes sabem, desde o início, que a terapia tem um limite de tempo definido.

Alguns preditores de abandono apontados na literatura foram identificados neste estudo: pais mais jovens e menos escolarizados tendem a abandonar o tratamento antes da alta (Campbell et al., 2000; Kazdin et al., 1997; Luk et al., 2001), assim como famílias mais expostas ao estresse (Kazdin, 1994; Prinz \& Miller, 1994; Sirles, 1990). Entre os estressores associados ao risco para abandono, destacaram-se os problemas financeiros (Garcia \& Weisz, 2002; Harwood \& Eyberg, 2004; Kazdin et al., 1997; Prinz \& Miller, 1994), e as situações sinalizadoras de conflito conjugal (Prinz \& Miller, 1994).

Attride-Stirling et al. (2004), comentando as diferenças entre famílias que persistem no tratamento até a alta e famílias que o abandonam, lembram a possibilidade de que estes dois grupos tenham buscado o tratamento por razões muito diferentes. As famílias que persistem podem estar focalizadas mais nas dificuldades da criança e então respondem a uma abordagem terapêutica focalizada na criança. Inversamente, as que abandonam o tratamento chegam com múltiplos problemas em diferentes níveis e então não são adequadamente servidas pela abordagem do atendimento com foco na criança. É importante compreender as necessidades e perspectivas dessas famílias, assim como um possível impacto, em seu funcionamento diário, de uma sobrecarga adicional, configurada pelo compromisso de comparecer semanalmente à clínica. Nesse contexto, é razoável sugerir que maior esforço deve ser feito para identificar e diferenciar casos com probabilidade de se beneficiarem de uma abordagem focada na criança, e casos que demandariam ajuda adicional em diferentes aspectos.

O abandono também foi associado a um desempenho mais pobre da criança no Raven. Este achado conflita com os do estudo de Boggs et al. (2004), que não encontraram associação entre medidas cognitivas e abandono em crianças. Porém é um resultado concordante com os de Kazdin et al. (1995). Em se tratando de um atendimento de apoio psicopedagógico para o enfrentamento de dificuldades escolares, as crianças mais prejudicadas talvez respondam mais lentamente à intervenção, o que pode ter levado a família a não ter suas expectativas 
supridas em relação à efetividade do tratamento, interrompendo-o precocemente.

Nas comparações entre os grupos, não se encontrou associação entre composição familiar e tipo de desfecho do atendimento. Essa ausência de associação está de acordo com os achados de Boggs et al. (2004), Dierker et al. (2001) e Lai et al. (1998).

No conjunto dos resultados, apenas duas dentre as 14 variáveis investigadas foram associadas à desistência e sete foram associadas ao abandono. Nesse aspecto de diversidade de preditores, os resultados deste estudo se assemelham àqueles obtidos em clínicas da comunidade, onde, conforme apontam Pina et al. (2003), têm sido encontrados menos preditores de desistência e abandono, em comparação aos obtidos em clínicas vinculadas a universidades, com amostras mais homogêneas. Embora nosso estudo tenha sido conduzido em uma clínica-escola, sua vinculação à rede de saúde provavelmente lhe confere características de funcionamento de um serviço comunitário, conforme comentado em parágrafo precedente.

Apesar de os estudos conduzidos em serviços comunitários apresentarem menor controle de variáveis, eles são mais condizentes com a realidade das instituições. Do mesmo modo que o cuidado clínico no mundo real difere em muitos aspectos do tratamento feito como parte de um protocolo de pesquisa, é possível que mesmo o processo de decisão e outros determinantes de interrupção do tratamento também sejam diferentes nos dois contextos (Garcia \& Weisz, 2002).

O estudo confirmou a importância de considerarem os diferentes momentos do atendimento, pois o grupo Desistente, comparado ao Não Desistente, e o grupo Abandono, comparado ao Grupo Alta Clínica, apresentaram perfis diversos de condições associadas à interrupção do vínculo com a clínica, confirmando sua heterogeneidade quanto ao desfecho (Marturano et al., 1993).

\section{CONCLUSÃO}

Vale considerar que neste trabalho investigaramse apenas os dados obtidos na primeira consulta. Não se teve acesso a fatores de risco que possam ter emergido após este momento. Em pesquisas futuras seria interessante contatar diretamente as famílias com o intuito de levantar, entre outros, fatores motivacionais e de satisfação em relação ao atendimento.

É importante também considerar que os resultados encontrados foram comparados aos resultados encontrados em estudos cuja origem predominante é norte-americana. Observa-se uma carência de estudos brasileiros que abordem o fenômeno da desistência e do abandono do atendimento de crianças.

Caracterizar as famílias que buscam o serviço é fundamental para o planejamento de ações visando prevenir possíveis desistências e abandonos e, assim, possibilitar o engajamento das mesmas durante o processo de atendimento. Ao conhecer melhor a clientela, pode-se adequar o atendimento e torná-lo mais efetivo para atender a demanda, contribuindo, dessa forma, para o aumento da taxa de adesão ao tratamento. Conseqüentemente, tanto as crianças, quanto suas famílias podem ser beneficiadas com a continuidade do tratamento até a alta clínica.

Como este estudo demonstrou, famílias que abandonam precocemente o suporte psicopedagógico estão enfrentando maior acúmulo de situações adversas, quando comparadas a famílias que persistem até a alta. Nesse contexto, a busca de ajuda psicológica adquire um significado mais amplo, que transcende a queixa escolar. É possível que essas famílias tenham expectativas de receber apoio para lidar com os estressores da vida cotidiana, entre os quais se incluem as dificuldades escolares e comportamentais da criança. A identificação precoce, já na entrevista de acolhimento, dos estressores psicossociais incidindo sobre a família ajuda a identificar as famílias em risco de interrupção do atendimento; essa informação pode ser o ponto de partida para práticas de suporte à família que, agregadas ao trabalho psicopedagógico junto à criança, provavelmente contribuirão para a redução do risco de abandono.

\section{REFERÊNCIAS}

Attride-Stirling, J., Davis, H., Farrell, C. G. \& Day, C. (2004). Factors influencing parental engagement in a community child and adolescent mental health service: A qualitative comparison of completers and non-completers. Clinical Child Psychology and Psychiatry, 9(3), 347-361.

Braga, S. G. \& Morais, M. L. S. (2007). Queixa escolar: atuação do psicólogo e interfaces com a educação. Psicologia USP, 18(4), 35-51.

Betti, M. C. M. (2007). Procura de atendimento psicológico por dificuldades escolares: um estudo das características da criança e seu ambiente familiar. Dissertação de Mestrado não publicada, Programa de Pós-Graduação em Psicologia, Universidade de São Paulo, Ribeirão Preto, SP.

Boggs, S. R., Eyberg, S. M., Edwards, D. L., Rayfield, A., Jacobs, J., Bagner, D. \& Hood, K. K. (2004). Outcomes of parent-child interaction therapy: a comparison of treatment completers and study dropouts one to three years later. Child \& Family Behavior Therapy, 26(4), 1-22. 
Campbell, V. A., Baker, D. B. \& Bratton, S. (2000). Why do child drop-out from play therapy? Clinical Child Psychology and Psychiatry, 5(1), 133-138.

Campezzato, P. V. M. \& Nunes, M. L. T. (2007). Atendimento em clínicas-escola de psicologia da região metropolitana de Porto Alegre. Estudos de Psicologia (Campinas), 24(3), 363-374.

D’Abreu, L. C. F. (2008). Identificação de problemas de saúde mental associados à queixa escolar segundo o DAWBA. Dissertação de Mestrado não publicada, Programa de PósGraduação em Saúde Mental, Universidade de São Paulo, Ribeirão Preto, SP.

Del Prette, Z. A. P. \& Del Prette, A. (2005). Psicologia das habilidades sociais na infância: teoria e prática. Petrópolis: Vozes.

Dierker, L., Nargiso, J., Wiseman, R. \& Hoff, D. (2001). Factors predicting attrition within a community initiated system of care. Journal of Child and Family Studies, 10 (3), 367-383.

Elias, L. C. S. \& Marturano, E. M. M. (2005). Oficinas de linguagem: proposta de atendimento psicopedagógico para crianças com queixas escolares. Estudos de Psicologia (Natal), 10(1), 53-61.

Garcia, J. A. \& Weisz, J. R. (2002). When youth mental health care stops: therapeutic relationship problems and other reasons for ending youth outpatient treatment. Journal of Consulting and Clinical Psychology, 70(2), 439-443.

Harpaz-Rotem, I., Leslie, D. \& Rosenheck, R. A. (2004).Treatment retention among children entering a new episode of mental health care. Psychiatric Services, 55(9), $1022-1028$

Harwood, M. D. \& Eyberg, S. M. (2004). Therapist verbal behavior early in treatment: relation to successful completion of parent-child interaction therapy. Journal of Clinical Child and Adolescent Psychology, 33(3), 601-612.

Kazdin, A. E. (1994). Adversidad familiar, desventajas socioeconomicas y estres en los padres: variables contextuales relacionadas con la termination prematura de la terapia conductual infantil. Psicología Conductual, 2(1), 5-21.

Kazdin, A. E., Holland, L. \& Crowley, M. (1997). Family experience of barriers to treatment and premature termination from child therapy. Journal of Consulting and Clinical Psychology, 65(3), 453-463.

Kazdin, A. E., Stolar, M. J. \& Marciano, P.L. (1995). Risk factors for dropping out of treatment among white and black families. Journal of Family Psychology, 9(4), 402417.

Kendall, P. C. \& Sugarman, A. (1997). Attrition in the treatment of childhood anxiety disorders. Journal of Consulting and Clinical Psychology, 65(5), 883-888.

Lai, K. Y. C., Pang, A. H. T., Wong, C. K., Lum, F. \& Lo, M. K. (1998). Characteristics of dropouts from a child psychiatry clinic in Hong Kong. Social Psychiatry \& Psychiatric Epidemiology, 33(1), 45-48.
Lau, A. S. \& Weisz, J. R. (2003). Reported maltreatment among clinic - referred children: Implications for presenting problems, treatment attrition, and long - term outcomes. Journal of the American Academy of Child and Adolescent Psychiatry, 42(11), 1327 - 1334.

Lhullier, A. C., Nunes, M. L. T. \& Horta, B. L. (2006). Preditores de abandono de psicoterapia em pacientes de clínica-escola. Em E. F. M. Silvares (Org). Atendimentos psicológicos em clínica-escola (pp. 229-246). Campinas: Alínea.

Luk, E. S. L., Staiger, P. K., Mathai, J., Wong, L., Birleson P. \& Adler, R. (2001). Children with persistent conduct problems who dropout of treatment. European Child \& Adolescent Psychiatry, 10(1), 28-36.

Marturano, E. M., Degani, I. C. C., Alves, C. X. \& Miranda, C. C. (1993). Abandono do atendimento psicopedagógico em um ambulatório de psicologia infantil ligado à Universidade. Medicina Ribeirão Preto, 26(2), 114-127.

Pina, A. A., Silverman, W. K., Weens, C. F., Kurtines, W. M. \& Goldman, M. L. (2003). A comparison of completers and noncompleters of exposure-based cognitive and behavioral treatment for phobic and anxiety disorders in youth. Journal of Consulting and Clinical Psychology, 71(4), 701705.

Prinz, R. J. \& Miller, G. E. (1994). Family - based treatment for childhood antisocial behavior: experimental influences on dropout and engagement. Journal of Consulting and Clinical Psychology, 62(3), 645-650.

Romaro, R. A. \& Capitão, C. G. (2003). Caracterização da clientela da clínica-escola de psicologia da Universidade São Francisco. Psicologia: Teoria e Prática, 5(1), 111-121.

Schoen-Ferreira, T. H., Silva, D. A., Aznar Farias, M. \& Silvares, E. F. M. (2002). Perfil e principais queixas dos clientes encaminhados ao Centro de Atendimento e Apoio Psicológico ao Adolescente (CAAA) - UNIFESP/EPM. Psicologia em Estudo, 7, 73-82.

Sirles, E. A. (1990). Dropout from intake, diagnostics, and treatment. Community Mental Health Journal, 26, 345-360.

Vanni, M. G. \& Maggi A. (2005). O que demanda à Psicologia na Rede Pública de Saúde em Caxias do Sul? Psico, 36(3), 299-309.

Wierzbicki, M. \& Pekarik, G. (1993). A meta-analysis of psychotherapy dropout. Professional Psychology: Research and Practice, 24(2), 190-195.

Recebido em 24/10/2008 Aceito em 14/06/2010
Endereço para correspondência:
Carina Cella Panaia Mantovani. Departamento de Neurociências e Ciências do Comportamento. Faculdade de Medicina de Ribeirão Preto - FMRP-USP. Rua Tenente Catão Roxo, 2650-CEP: 14051-140. Ribeirão Preto, S.P, Brasil. E-mail: mantovani.clinica@gmail.com 